Homer CSE, Johnson R, Foureur M. (2011). Next birth after caesarean section: Changes over a nine-year period in one Australian state. Midwifery 27 (2) 165-169.

\title{
BIRTH AFTER CAESAREAN SECTION: CHANGES OVER A NINE-YEAR PERIOD IN ONE AUSTRALIAN STATE
}

\begin{abstract}
Objectives: The aim of the study was to describe the outcomes related to birth after a caesarean section (CS) in one Australian state, New South Wales (NSW), over a nine year period. The objectives were to determine whether changes had occurred in the rates of attempted and successful vaginal birth after caesarean section (VBAC), induction of labour, place of birth, admission to special care or neonatal intensive care nursery and perinatal mortality.
\end{abstract}

Design and setting: Cross-sectional analytic study of hospital births in New South Wales using population-based data from 1998-2006.

Participants: Women experiencing the next birth after a CS where: the total number of previous CS was 1; the presentation at birth was vertex; it was a singleton pregnancy; and, the estimated gestational age was greater than or equal to 37 weeks. A total of 53,455 women met these criteria.

Measurements: Data were obtained from NSW Health Department's Midwives Data Collection (MDC). The MDC includes all live births and stillbirths of at least 20 weeks gestation or $400 \mathrm{~g}$ birth weight in the state.

Findings: Over the nine year period, the rate of vaginal birth after caesarean section (VBAC) declined significantly (31\% to 19\%). The proportion of women who 'attempted a vaginal birth' also declined (49\% to 35\%). Of those women who laboured, the vaginal birth rate declined from (64\% to 53\%). Babies whose mothers 'attempted' a VBAC were significantly less likely to require admission to a special care nursery (SCN) or neonatal intensive care. The perinatal mortality rate in babies whose mothers 'attempted' a VBAC was higher than those babies born after an elective caesarean section although the absolute numbers are very small.

Key conclusions: Rates of VBAC have declined over this nine year period. Rates of neonatal mortality and proxy measures of morbidity (admission to a nursery) are generally in the low range for similar settings.

Implications for practice: Decisions around the next birth after CS are complex. Efforts to keep the first birth normal and support women who have had a CS to have a normal birth need to be made. More research to predict which women are likely to achieve a successful VBAC and the most effective ways to facilitate a VBAC is essential. Midwives have a critical role to play in these endeavours. 


\section{NEXT BIRTH AFTER CAESAREAN SECTION: CHANGES OVER A NINE-YEAR PERIOD IN ONE AUSTRALIAN STATE}

\section{INTRODUCTION}

Caesarean section (CS) is becoming increasingly common, especially in developed countries. In the United States of America (USA) it is the most common surgical procedure performed on women (Sheppard and Tumarkin, 2005). In many countries, the rate of CS has risen considerably in recent years without any apparent improvement in maternal and fetal wellbeing. In Australia, the increase in CS has been dramatic, from 19.5\% in 1996 (Day et al. 1999) to 30.8\% in 2006 (Laws and Hilder, 2008). In England, Scotland and Wales the rates increased from $16-17 \%$ in 1995 to $23-25 \%$ in 2005, an increase again of around 50\% (Maternity Care Working Party, 2007). Similarly, in the USA, caesarean births have increased by 50\% in the past decade, from 20.7\% in 1996 to 31.1\% in 2006 (MacDorman et al. 2008). An increasing CS rate is also a global concern, with high rates in many developing countries as well (Belizan et al. 1999).

It has been hypothesised by researchers in the USA, that the increase in the CS rate reflects two concurrent trends: an increase in the primary cesarean rate and a decline in the vaginal birth after CS (VBAC) rate (MacDorman et al. 2008). It is likely that this is a similar situation in other developed countries where decisions around the next birth after caesarean section are often challenging for women and clinicians.

Concerns about the safety of VBAC have influenced practice around the next birth after CS for more than three decades. The issue is whether or not the uterine scar will rupture during the course of labour and vaginal birth resulting in injury or even death of mother or baby. Women are often advised that a repeat caesarean is the best option or conversely that VBAC is a safe and effective choice. Women in other settings are either denied the choice of attempting a vaginal birth or are persuaded that choosing it is dangerous. There is often a conflict of advice for women in this area. Obtaining accurate data is a high research priority in order that women and their clinicians can make truly informed choices about appropriate birth options.

The aim of the study was to describe the outcomes related to the next birth after a caesarean section (CS) in one Australian state, New South Wales (NSW) over a nine year period. The objectives were to determine whether changes had occurred in the rates of attempted and successful VBAC, induction of labour, place of birth, admission to special care or neonatal intensive care nursery and perinatal mortality. NSW is the largest Australian State in terms of population and number of births. In 2006, NSW had one third of all births in Australia (277,436 births in Australia of which 91,303 were in NSW). It was therefore seen as important to measure the changes in this Australian state.

\section{METHODS}

Data were obtained from NSW Health Department's Midwives Data Collection (MDC). The MDC includes all live births and stillbirths of at least 20 weeks gestation or $400 \mathrm{~g}$ birth weight that occur in the state, and includes information on maternal demographic factors, pregnancy, labour, delivery and perinatal outcomes. 
The population included all women whose last birth was by caesarean section. The NSW Health Department Centre for Epidemiology and Research provided the data and these have been used under conditions specified by the providers of the data. The data provided are classified as re-identifiable data by Australia's National Health and Medical Research Council (NHMRC) (NHMRC, 2007), that is, the identifiers have been removed and replaced by a code. It remains possible to re-identify a specific individual by, for example, using the code or linking different data sets however the researchers did not have access to such a capacity and so the data for the purposes of this study are unidentifiable. Ethical approval for this study was therefore not required in line with NHMRC guidelines (NHMRC 2007).

Eligibility for the study required that: the last birth was by caesarean section; the total number of previous caesarean sections was 1 ; the presentation at birth was vertex; there was a singleton pregnancy; and, the estimated gestational age was greater than or equal to 37 weeks.

The MDC database does not identify whether women chose to labour in an effort to have a VBAC. The data identifies which women laboured, either spontaneously or after an induction of labour. These variables identify attempting a VBAC and provide a surrogate marker for 'choosing' a VBAC. The rate of attempted VBAC was calculated by selecting women who were 'eligible' for a VBAC. Two rates of vaginal birth (that is, successful VBAC) were calculated. Firstly, the rate of vaginal birth (including vacuum extraction and forceps) for women who were 'eligible' was calculated (that is, the overall VBAC rate). Secondly, the rate of vaginal birth (including vacuum extraction and forceps) for women who had actually had an onset of labour (either spontaneous or induced) was calculated (that is, VBAC rate in women who 'chose' a VBAC).

Other analyses included the rate of successful VBAC in women who have had a previous vaginal birth compared with women who have not; the outcomes for women who had an induced labour compared with a spontaneous labour; and, the place of birth: birth centre or homebirth compared with conventional labour ward. All these analyses were undertaken by year to assess trends over time.

The neonatal outcome measures were: stillbirth or neonatal death; and, admission to a neonatal intensive care unit (NICU) or SCN (special care nursery). Mothers with a fetal death in utero diagnosed prior to the onset of labour were excluded. Neonatal outcomes were analysed according to maternal intention to have a VBAC, that is, attempted VBAC versus elective CS.

Crude odds ratios and 95\% confidence intervals (95\% CI) were produced for each outcome. An odds ratio is a measure of effect size, describing the strength of association or nonindependence between two binary data values. It is used as a descriptive statistic. The chi squared $\left(\chi^{2}\right)$ test for linear trend was calculated to assess changes over the nine year period.

\section{FINDINGS}

During the study period a total of 788,810 women gave birth in NSW. Of these, 53,455 $(6.78 \%)$ met the inclusion criteria for this study. Figure 1 presents the trajectory of the sample in terms of onset of labour and mode of birth.

$<$ Insert Figure 1 here $>$ 
The rate of vaginal birth for women with a previous caesarean section declined significantly from 31\% in 1998 to $19 \%$ in 2006 ( $\chi^{2}$ for linear trend $=282.3(1 \mathrm{DF}) \mathrm{P}<0.0001$ ) (Figure 2).

$<$ Insert Figure 2 here $>$

The proportion of women who commenced labour, either spontaneously or through an induction of labour, had declined significantly over the nine year period. In 1998, almost half (49\%) of women with one previous caesarean section at term, a singleton pregnancy and a vertex presentation commenced labour compared with just over one third (35\%) in $2006\left(\chi^{2}\right.$ for linear trend $=43.2(1 \mathrm{DF}) \mathrm{P}<0.0001)$. Of the women who commenced labour, the rate of vaginal birth has also decreased significantly over the study period. In 1998, the rate of vaginal birth in women who actually commenced labour was $64 \%$ compared with $53 \%$ in 2006 ( $\chi^{2}$ for linear trend $\left.=746.0(1 \mathrm{DF}) \mathrm{P}<0.0001\right)$.

Of the women who had an onset of labour, 18\% were induced. Labour was induced using prostaglandins (7.1\%), oxytocics (16.9\%), through an artificial rupture of membranes $(11.0 \%)$ or by other mechanisms, for example, using an intracervical catheter (3.4\%). The use of prostaglandins decreased significantly in the nine year period, from 44 women in 1998 to only one woman in 2006.

The rate of vaginal birth for women who underwent induction of labour compared with those who had a spontaneous onset of labour was calculated. The vaginal birth rate for women who commenced labour spontaneously declined significantly from 64\% in 1998 to 54\% in 2006 ( $\chi^{2}$ for linear trend $\left.=142.15(1 \mathrm{DF}) \mathrm{P}<0.0001\right)$ as did the rate for women who had an induction of labour. This latter group's rate declined from 65\% in 1998 to 50\% in $2006\left(\chi^{2}\right.$ for linear trend $=23.91(1 \mathrm{DF}) \mathrm{P}<0.0001)$. The overall vaginal birth rate for women who spontaneously laboured was $59 \%$ and $62 \%$ for women who were induced. The women who were induced were more likely to have a vaginal birth than those who laboured spontaneously (OR 1.13; 95\% CI 1.05-1.21).

The outcomes for women who had a previous vaginal birth prior to the caesarean section were examined. The proportion of these women going on to have a vaginal birth declined significantly from 83\% in 1998 to $70 \%$ in $2006\left(\chi^{2}\right.$ for linear trend $=37.3$ (1 DF) $\mathrm{P}<$ $0.0001)$. Women with a previous vaginal birth were almost 3 times more likely to have a vaginal birth compared with women who had not (OR 2.98; 95\% CI 2.76-3.22).

Finally, planned place of onset of labour was examined. Two categories were used: hospital labour ward/delivery suite or birth centre which included a small number of planned homebirths. Women who had an induction of labour were excluded from the labour ward group for the purposes of this comparison as women do not usually undergo induction of labour in a birth centre setting. The numbers of women planning to give birth in a birth centre or at home was small - 360 in total over nine years (between 28 and 57 women annually). The rate of vaginal birth decreased significantly in the labour ward group (63\% to 53\% $\left(\chi^{2}\right.$ for linear trend $=41.9(1 \mathrm{DF}) \mathrm{P}<0.0001)$ but had not changed significantly in the birth centre group over the nine year period (Figure 3).

$<$ Insert Figure 3 here $>$

\section{Neonatal morbidity and mortality}


Data were available for 53,450 babies (data was missing for 4 babies). Babies diagnosed as a fetal death in utero prior to the onset of labour were excluded from the analysis of perinatal mortality ( $n=29)$. This left 53,421 babies for analysis. Admission to a NICU or SCN and perinatal mortality were analysed according to 'intention to have a VBAC' (that is, attempted) versus 'an elective repeat CS' over time.

The admission to NICU or SCN had altered little over time ranging between 6-8\% per year. Babies whose mothers 'attempted' a VBAC were significantly less likely to require admission to a special care nursery (SCN) or neonatal intensive care (NICU) (OR 0.89; 95\% CI 0.83-0.97).

In total, 67 babies died in this cohort giving a perinatal mortality rate of 1.25 per 1000 births. The perinatal mortality rate ranged from 0 to 3.28 over the nine year period (Table 1). The perinatal mortality rate in babies whose mothers 'attempted' a VBAC was higher than those babies born after an elective caesarean section (OR 1.79; 95\% CI 0.1.08-2.98).

\section{$<$ Insert Table 1 here $>$}

\section{DISCUSSION}

This population-based study of all women giving birth in one Australian state over a nine year period has demonstrated significant changes in relation to uptake of VBAC and success at achieving a vaginal birth. The main changes have been the reduction in women seeking or attempting to have a vaginal birth after a previous caesarean section and decreased success in achieving this outcome.

Internationally, VBAC rates have decreased in the recent years. For example, in the US, from 1996 to 2004, the VBAC rate decreased from 28.3\% to 9.2\% with a corresponding increase in the rate of primary CS (MacDorman et al. 2008). It has been suggested that the decrease is due to changes of emphasis in US guidelines on VBAC (Roberts et al. 2007). In 1999, the American College of Obstetricians and Gynecologists issued a practice bulletin that recommended, "Most women with one previous cesarean delivery with a low-transverse incision are candidates for VBAC and should be counseled about VBAC and offered a trial of labor'”. The bulletin tempered this by recommending that surgical capability be “immediately available” for women in labour attempting VBAC (American College of Obstetricians and Gynecologists, 1999). This new recommendation was based on category $C$ evidence (consensus opinion). A recent review of 314 hospitals in four states in the USA showed that almost one-third of hospitals that previously offered VBAC services had stopped doing so. Of the hospitals that continued to offer VBAC services, 68 percent had changed their VBAC policies since 1999, most particularly the need for immediate access to anesthesia when women desiring VBAC presented in labor (Roberts et al. 2007). In the United Kingdom (UK), the National Caesarean Section Sentinel Audit showed that repeat CS contributed 29\% to the overall CS rate. In the Audit, the overall VBAC rate was 33\% with a range of 27-38\% between regions and 6-64\% between units (Thomas and Paranjothy, 2001). Other reasons likely to be contributing to the changes over time in this setting include fear of normal labour and birth from some women and clinicians, uncertainly about the safety of VBAC with related restrictive policies about eligibility and the management of labour and fear of litigation. More research is needed to 'unpick' these issues and others in relation to their contribution to the changes over time in VBAC rates. 
Severe maternal morbidity is clearly an important outcome to consider when determining whether VBAC should be offered to all women. A recent meta-analysis from the United States (US) reviewed maternal morbidity following trial of labour after cesarean section, compared with elective repeat cesarean delivery (Rossi and D'Addario, 2008). The successful VBAC rate was $73 \%$ with rates of maternal morbidity, blood transfusion and hysterectomy similar in women planning VBAC or elective repeat CS. Women planning VBAC had a higher rate of uterine rupture/dehiscence $(1.3 \%$; 0,4\%). Maternal morbidity was lower in women who had successful VBAC than an elective repeat cesarean delivery. The study concluded by saying that while there was a higher risk of uterine rupture/dehiscence in planning a VBAC, this should be balanced against a reduction in maternal morbidity when VBAC is successful. Clearly this study highlights the need to identify better ways to predict successful VBAC.

In our study, women who were induced were more likely to have a vaginal birth than those who laboured spontaneously. This could be because women who were assessed to have a favourable cervix were encouraged to try for a VBAC whereas women with unfavourable cervices were encouraged to have a repeat CS. In general, there are concerns about induction of labour in women with a previous CS. A number of studies demonstrate that induction of labour is associated with higher rates of uterine rupture or dehiscence (RCOG, 2007). The method for inducing labour has also changed over time in NSW. In particular, the use of prostaglandins had decreased significantly in the nine year period, from 44 women in 1998 to only one woman in 2006. This is in line with the literature suggesting concerns around the use of prostaglandins in women with a previous CS (Goldberg et al. 2001).

Our study could not determine which women would have preferred to have a VBAC. We have used labour as a surrogate marker for choosing or preferring to have a vaginal birth. The MDC data system does not collect preference of the woman towards model of birth. In other studies, it is suggested that many women with a previous CS prefer a vaginal birth for the next birth. For example, an analysis of the records of more than 200 women at a London hospital demonstrated that, VBAC was the method of delivery preferred by most women although only $37.8 \%$ of those who chose it were delivered by it (Selo-Ojeme et al. 2008). In Australia, a qualitative study showed that women held strong views about the importance of working with their bodies to achieve a vaginal birth after a prior caesarean section (Fenwick et al. 2007). Women in the study were able to articulate the risks of caesarean but considered that vaginal birth enhanced their health and well-being and that of their baby's. In the USA, the Listening to Mothers survey, conducted in 2002, found little interest in a future elective primary cesarean (Declercq et al. 2002). The survey also showed that the willingness of caregivers to 'permit;' a VBAC had dramatically reduced in women who had given birth in the previous 12 months compared with women who had given birth before 12 months further supporting the impact that the 1999 ACOG VBAC Practice Bulletin (American College of Obstetricians and Gynecologists, 1999) had on choice and access. In future research, it would be interesting to select a random sample of this study population to explore their decision making process.

Our study is retrospective using existing data sets and we were not able to determine the uterine rupture rate over time. These data are not collected routinely in this data set and data linkage would be required to determine this. We recognise this as a limitation. An earlier Australian study reported the risk of uterine rupture in a second pregnancy following caesarean section in the first pregnancy during the time period 1988-2002 (Taylor et al. 2005). Only 20 cases of uterine rupture were found among mothers who underwent labour 
over this 5-year period, 11 in the group with a primary CS and 9 in the group who had not. The overall uterine rupture rate in women with a prior primary CS was 0.54 per 1000 . The authors concluded that, while primary caesarean section (compared with primary vaginal birth) conferred additional risk of complications in the second pregnancy for mother, complications were uncommon, regardless of whether the mother had a primary caesarean section.

The Taylor et al (2005) study is important as it considered the number of women who need to give birth to produce one additional bad outcome - the 'number needed to harm'. The additional risk conferred by a primary caesarean section was 0.04 per 1000 births for uterine rupture in labour which translates to requiring 25,000 additional caesarean sections for the 'harmful' outcome of uterine rupture in labour. Clinically, this information is useful as it highlights the rarity of the most adverse outcomes, an understanding that may have been overlooked in recent years as seen by the reduction in VBAC rates - both being offered and being successful. It is likely that a substantial element of fear has crept into decision making around VBAC and the rarity of adverse outcomes has been forgotten.

The perinatal mortality rate in our study was low (0.95 per 1000$)$ although higher in the group who attempted a VBAC (1.7 per 1000). The women who were induced for a known antenatal still birth were excluded from this analysis although the dataset does not enable removal of babies who had severe congenital abnormalities and were expected not to live. As the overall numbers of deaths is small ( 37 vs 30 ) it is possible that some of the deaths in the attempted VBAC group were in women whose babies were not expected to live. It may also be that a subset of the least healthy babies were stressed by labour but corresponding babies in the elective caesarean group survived the experience to be born alive but unwell. This requires further research to explain adverse outcomes. The higher perinatal mortality rate is tempered by a lower morbidity, as measured by lower admissions to special care and neonatal intensive care. Research to better understand neonatal morbidity associated with birth after CS is also needed.

\section{CONCLUSION}

This study contributes to the understanding of what has occurred to VBAC over time in one country and may have resonance for other similar countries. The study demonstrated significant changes in the uptake, and success of, VBAC over a nine year period. It is likely that similar patterns are being seen in many developing countries as fears around adverse events play out into practice settings. Influences include changes in guidelines and a possible lack of commitment from clinicians in relation to supporting VBAC as the norm rather than the exception.

As national CS rates continue to rise, or remain high, significant efforts need to be made into keeping women's first birth normal and supporting women who have had a CS to have a normal birth. More research to predict which women are likely to achieve a successful VBAC and the most effective ways to support women to have a VBAC is essential. Midwives have a critical role to play in these endeavours. 


\section{REFERENCES}

American College of Obstetricians and Gynecologists. 1999. Birth after Cesarean Delivery. ACOG Practice Bulletin, No. 5, July 1999 (replaces Practice Bulletin No. 2, October 1998). Journal of Gynaecology and Obstetrics 66, 197-204.

Belizan J, Althabe F, Barros F, Alezander S. 1999. Rates and implications of caesarean sections in Latin America: ecological study. BMJ 319, 1397-402.

Day, P., Sullivan, E., Lancaster, P. 1999. Australia's Mothers and Babies 1996. Australian Institute of Health and Welfare, Canberra.

Declercq, E.R., Sakala C., Corry, M.P., Applebaum, S., Risher, P. 2002. Listening to Mothers: Report of the First National U.S. Survey of Women's Childbearing Experiences. Maternity Center Association, New York,

Fenwick, J., Gamble, J., Hauck, Y. 2007. Believing in birth - choosing VBAC: The childbirth expectations of a self-selected cohort of Australian women. Journal of Clinical Nursing 16(8), 1561-70.

Goldberg, A.B., Greenberg, M.A., Darney, P.D. 2001. Drug therapy: Misoprostol and pregnancy. New England Journal of Medicine 344(1), 38-47.

Laws, P., Hilder, L. 2008. Australia's mothers and babies. Australian Institute of Health and Welfare, Canberra.

MacDorman, M.F., Menacker, F., Declercq, E. 2008. Cesarean birth in the United States: epidemiology, trends, and outcomes. Clinics in Perinatology 35(2), 293-307.

Maternity Care Working Party. 2007. Making normal birth a reality. Consensus statement from the Maternity Care Working Party. NCT/RCM/RCOG, London.

National Health and Medical Research Council. 2007. National Statement on Ethical Conduct in Human Research. Australian Government, Canberra.

RCOG. 2007. Birth after previous caesarean birth: Green-top Guideline No. 45 London: Royal College of Obstetricians and Gynaecologists, London.

Roberts, R.G., Deutchman, M., King, V.J., Fryer, G.E., Miyoshi, T.J. 2007. Changing policies on vaginal birth after cesarean: impact on access. Birth 34(4), 316-22.

Rossi, A.C., D'Addario, V. 2008. Maternal morbidity following a trial of labor after cesarean section vs elective repeat cesarean delivery: a systematic review with meta-analysis. American Journal of Obstetrics \& Gynecology 199(3), 224-31.

Selo-Ojeme, D., Abulhassan, N., Mandal, R., Tirlapur, S., Selo-Ojeme, U. 2008. Preferred and actual delivery mode after a cesarean in London, UK. International Journal of Gynaecology \& Obstetrics 102(2), 156-9. 
Sheppard, J., Tumarkin L. 2005. A Mother's Right to Know: New York City Hospitals Fail to Provide Legally Mandated Maternity Information. New York, Office of the New York City Public Advocate, New York.

Taylor, L., Simpson, J., Roberts, C.L., Olive, E.C., Henderson-Smart, D.J. 2005. Risk of complications in a second pregnancy following caesarean section in the first pregnancy: a population-based study. Medical Journal of Australia 183, 515-519.

Thomas, J., Paranjothy S. 2001. National Sentinel Caesarean Section Audit Report. RCOG Press, London. 